\title{
PHOTON COLLIDERS IN PHYSICAL PROGRAM FOR LARGE COLLIDERS
} Talk given at Workshop on Electromagnetic Probes of Fundamental Physics, Eriche, Sicily, 16-21 October 2001

\author{
I.F. Ginzburg ${ }^{1}$, S.I. Polityko ${ }^{2}$ \\ ${ }^{1}$ Institute of Mathematics and Novosibirsk State University, Novosibirsk, Russia; \\ ${ }^{2}$ Irkutsk State University, Irkutsk, Russia
}

\begin{abstract}
In this report our main attention is focused on the problems which can be solved at Photon Colliders naturally but are hardly solved at LHC and $e^{+} e^{-}$Linear Collider: The New Physics - new particles, new interactions, supersymmetry, Dirac monopole, extra dimensions... SM and the case of a SM-like scenario - EWSB, Higgs bosons, anomalies in interactions of gauge bosons, QCD, Pomeron and odderon... By-product: Production of light Goldstone particles from region of $e \rightarrow \gamma$ conversion.
\end{abstract}

\section{Introduction}

Photon Collider will be a specific mode of Linear Collider (LC) obtained with the aid of laser light backscattering on the accelerated electrons in Linear Collider near the collision point $1 \mathrm{E}$. Let us enumerate its main characteristics in the frame of TESLA project $\mathrm{B}$.

1. Characteristic photon energy $E_{\gamma} \approx 0.8 E$ ( $E$ - energy of electron in the basic $e^{+} e^{-}$collider).

2. For high energy peak, $E_{\gamma 1,2}>0.7 E_{\gamma \max }$ (separated well from low energy part of spectrum)

- Luminosity $\mathcal{L}_{\gamma \gamma} \approx \mathcal{L}_{e e} / 3, \mathcal{L}_{e \gamma} \approx \mathcal{L}_{e e} / 4$ with $\int \mathcal{L}_{\gamma \gamma} d t, \int \mathcal{L}_{e \gamma} d t \approx 200 \div 150 \mathrm{fb}^{-1} /$ year.

- Mean energy spread $\left\langle\Delta E_{\gamma}\right\rangle \approx 0.07 E_{\gamma}$ (by factor $2 \div 3$ worse than in $e^{+} e^{-}$mode considering beamstrahlung and ISR).

- Mean photon helicity $\left\langle\lambda_{\gamma}\right\rangle \approx 0.95$, with easily variable sign. One can also get the linear polarization. (In the $e^{+} e^{-}$mode only longitudinal polarization is relevant.)

3. The $e \rightarrow \gamma$ conversion region is $e \gamma$ collider with $\sqrt{s_{e \gamma}} \approx 1.2 \mathrm{MeV}$ and $\mathcal{L} \sim 0.1 \mathrm{fb}^{-1} / \mathrm{sec}$ !

4. The total additional cost is estimated as about $10 \%$ from that of ${ }^{+} e^{-}$LC.

The Standard Model (SM) is verified now with high precision except for mechanism of electroweak symmetry breaking (EWSB). The new large colliders are constructed with the dream to find New Physics and understand EWSB mechanism. We discuss different physical pictures obtained after running of large colliders of next generation (Tevatron, LHC and LC):

1. Clear signals of New Physics (new particles, strong deviations from SM) will be found.

2. The physical picture will coincide with that expected in SM within experimental precision - SM-like scenario, determined for the time of observations:

- No new particles and interactions will be discovered except for single Higgs boson.

- The couplings of Higgs boson to $W, Z$ and quarks will coincide with those predicted in SM within the experimental precision.

We discuss the potential of Photon Collider in both these scenarios separately. For more details on some mentioned points see refs. 腘. 


\section{Hunting for New Physics}

\subsection{Discovery of new particles}

The discovery of a new particle will be a clean signal of some definite form of a new theory. In the discussion below we denote the kinematically allowed discovery bound for its mass as $M_{b}$.

The production of pair of charged particles in $\gamma \gamma$ collisions near the threshold is described by QED with reasonable accuracy. The corrections due to other (even strong) interactions can be neglected in the estimates of opportunity to discover the particle. Therefore, the real discovery limit of new particle in $\gamma \gamma$ channel is close to $M_{b} \approx 0.8 E$, it is lower than that in ${ }^{+} e^{-}$mode.

The $e \gamma$ collisions provide us with final states which cannot be produced with similar intensity other ways. In reactions like $e \gamma \rightarrow B A$ with light particle $A$ and new particle $B$ the kinematical discovery bound $M_{b}$ can be much higher than in other reactions, $M_{b} \leq 1.8 E$. However, the cross section of such process depends on new coupling constants like $e A B$. Therefore - in contrast to $\gamma \gamma$ collisions - the absence of such signals can be explained by absence of new particle $B$ as well as by its "electrofobic" nature (very small $e A B$ coupling).

The $e \gamma$ mode provides the best opportunities for discovering a number of new particles. The discovery limits $M_{b}$ of some new states are presented in Table 1 . For the excited

\begin{tabular}{|c|c|c|c|c|c|}
\hline & & name & reaction & $M<M_{b}$ & observed \\
\hline & $e^{*}$ & excited $e$ & $e \gamma \rightarrow e^{*}$ & $1.8 \mathrm{E}$ & $e \gamma$ or $e Z$ \\
\cline { 2 - 6 } & $\nu_{e}^{*}$ & excited $\nu$ & $e \gamma \rightarrow W \nu_{e}^{*}$ & $1.8 E-M_{W}$ & $e W$ \\
\cline { 2 - 6 } & $W^{\prime}$ & new $W$ & $e \gamma \rightarrow \nu W^{\prime}$ & $1.8 \mathrm{E}$ & $W Z, W \gamma$ \\
\hline \hline \multirow{2}{*}{$\mathrm{S}$} & $\tilde{W}$ & wino & $e \gamma \rightarrow \tilde{W} \chi$ & $1.8 E-M_{\chi}$ & \\
\cline { 2 - 6 } $\mathrm{U}$ & $\tilde{Z}$ & zino & $e \gamma \rightarrow \tilde{Z} \tilde{e}$ & $1.8 E-M_{\tilde{e}}$ & \\
\cline { 2 - 6 } $\mathrm{S}$ & $\tilde{e}$ & selectron & $e \gamma \rightarrow \tilde{e} \chi$ & $1.8 E-M_{\chi}$ & \\
\cline { 2 - 6 } $\mathrm{Y}$ & \multicolumn{5}{|c|}{$\chi S P$, lightest superparticle } \\
\hline
\end{tabular}

Table 1: Some discovery limits for er mode

electron the expected cross section is high enough to observe it even with the weak enough coupling constant.

The $\gamma \gamma$ mode. Pair production. The cross section of the pair production $\gamma \gamma \rightarrow P^{+} P^{-}$ ( $P=S$ - scalar, $P=F$ - fermion, $P=W$ - gauge boson) not far from the threshold is given by QED as $\left(\lambda_{i}-\right.$ circular, $\ell_{i}-$ linear polarization of photons, $\left.\phi=\angle\left(\vec{\ell}_{1}, \vec{\ell}_{2}\right)\right)$

$$
\begin{gathered}
\sigma=\frac{\pi \alpha^{2}}{M_{P}^{2}} C_{q} f_{P}\left(\frac{s}{4 M_{P}^{2}}\right) \approx 65\left(\frac{1 T e V}{M_{P}}\right)^{2} C_{q} f_{P}\left(\frac{s}{4 M_{P}^{2}}\right) \mathrm{fb} \\
f_{P}(x) \equiv f_{P}^{0}(x)+\lambda_{1} \lambda_{2} g_{P}^{a}+\ell_{1} \ell_{2} \cos 2 \phi g_{P}^{\tau} ; \quad C_{q}=\left\{\begin{array}{cl}
1 & \text { if } \quad Q=1, \\
3 Q^{4} & \text { if } \quad Q \text { is noninteger }
\end{array}\right.
\end{gathered}
$$

(with functions $f_{P}^{0}, g_{P}^{a}$ and $g_{P}^{\tau}$ written e.g. in ref.15). These $f_{P}^{0}$ (for the unpolarized photons) are shown in Fig. $1\left(W^{2}=s\right)$. The $\gamma \gamma$ cross sections are evidently higher than the corresponding $e^{+} e^{-}$collisions, which are also shown here 中.

With expected luminosities the first discovery of new particle is preferable in $e^{+} e^{-}$mode. After that, the key problem will be to study the nature of the discovered particle. In this respect, the $\gamma \gamma$ production provides essential advantages compared to $e^{+} e^{-}$collisions:

- These cross sections decrease slow with energy growth. Therefore, one can study these processes relatively far from the threshold where the decay products don't overlap.

${ }^{a}$ The observable $e^{+} e^{-} \rightarrow P^{+} P^{-}$cross section includes also $Z^{*}$ contribution, dependent on other quantum numbers besides charge and spin. At $s \gg M_{Z}^{2}$ typically $\sigma\left(e^{+} e^{-} \rightarrow P^{+} P^{-}\right)<1.3 \sigma\left(e^{+} e^{-} \rightarrow \gamma^{*} \rightarrow P^{+} P^{-}\right)$. 

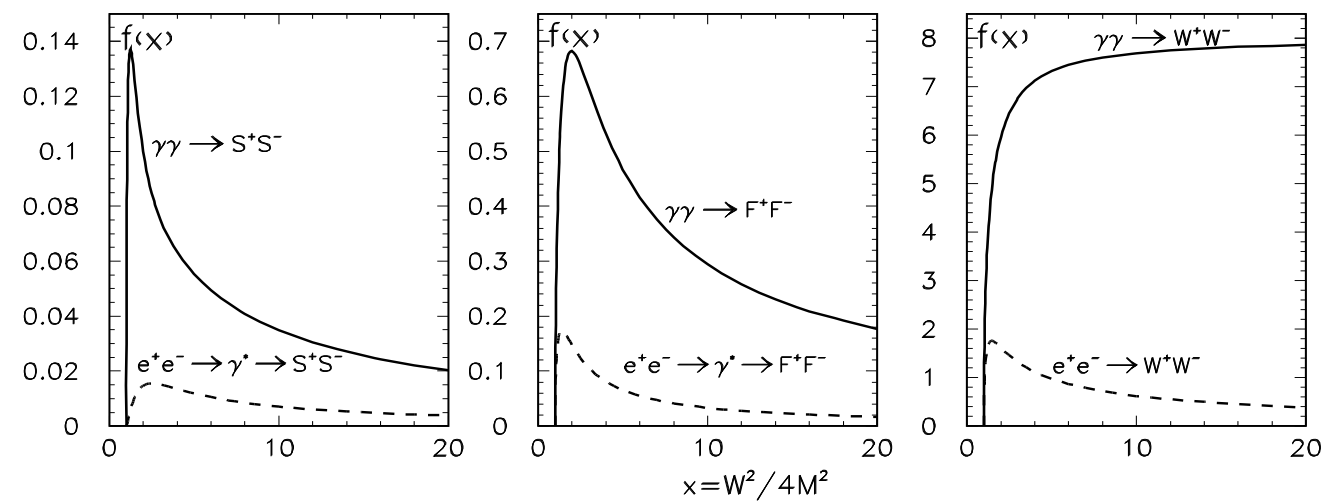

Figure 1: The $\gamma \gamma \rightarrow P^{+} P^{-}$cross sections, divided by $\left(\pi \alpha^{2} / M_{P}^{2}\right)$, nonpolarized photons, $C_{q}=1$. The cross sections $e^{+} e^{-} \rightarrow \gamma^{*} \rightarrow P^{+} P^{-}$are also shown.

- Near the threshold $f_{P} \propto\left(1+\lambda_{1} \lambda_{2} \pm \ell_{1} \ell_{2} \cos 2 \phi\right)$ with + sign for $P=S$ (scalar) and - sign for $P=F$ (fermion). This polarization dependence provides the opportunity to know spin of produced particle independent on its charge. (This problem arises, e.g., at the discovery of SUSY particles since spin of invisible neutral is unknown).

- The possible $\mathrm{CP}$ violation in the $P \gamma$ interaction can be seen as a variation of cross section with changing the sign of photon helicity.

The discovery of some other particles in $\gamma \gamma$ mode.

- The leptoquark $(\ell q)$ can be discovered in reactions like $\gamma \gamma \rightarrow \bar{\ell}+t+(\ell t)$ with $M_{b} \approx 1.5 E_{\gamma}$.

- The scalar or tensor resonances $R$ appearing due to the strong interaction in Higgs sector can be discovered in process $\gamma \gamma \rightarrow R$ with $M_{b} \approx 2 E_{\gamma}$.

- The gluino $\tilde{g}$ can be produced in process $\gamma \gamma \rightarrow \tilde{g} \tilde{g}$ (via quark loop) 5 . The maximal value of this cross section is $\sim\left(\alpha^{2} \alpha_{s}^{2} / M_{\tilde{q}}^{2}\right) \ln \left(M_{\tilde{q}}^{2} / M_{q}^{2}\right)$ at $2 M_{\tilde{g}}<\sqrt{s_{\gamma \gamma}}<2 M_{\tilde{q}}$. For example, this cross section is about $1 \mathrm{pb}$ at $M_{\tilde{q}}=0.5 \mathrm{TeV}, M_{\tilde{g}}=0.25 \mathrm{TeV}$ for $\sqrt{s_{\gamma \gamma}}=1.5 \mathrm{TeV}$.

- If the stop squark is not too heavy, the very narrow atom-like (scalar) stoponium with mass 200-400 GeV should also exist (such states cannot be observed at hadron collider). It can be clearly seen at $\gamma \gamma$ collider with cross section averaged over photon spectrum $<\sigma>\approx 10-50 \mathrm{fb}$ and clear enough signature 6 .

\subsection{Some problems with new interactions}

- Due to high values of the basic cross sections for the pair production of charged particles, Photon Collider would be an excellent place for observation of (even small) possible flavor changing neutral currents (FCNC), for example, with superparticles.

- In the 2HDM or MSSM in the CP conserving case the masses of heavy Higgs scalar $H$ and pseudoscalar $A$ can be close to each other or they are mixed (in the CP violated - $C \mathrm{P}$ - case). The observations of decay products at LHC and $e^{+} e^{-} \mathrm{LC}$ cannot resolve these opportunities due to low resolution for these bosons. The polarization asymmetries in Higgs boson production at Photon Collider can resolve these variants, i.e. establish, whether CP parity in Higgs sector is violated or not.

Using the $\gamma \gamma \rightarrow \gamma \gamma$, etc. processes for the search of effects from extra dimensions or heavy point-like Dirac monopole. In both cases the process is considered strongly below new mass scale $M$ or particle production threshold $2 M$. In both cases the cross section can be written as

$$
\sigma(\gamma \gamma \rightarrow \gamma \gamma)=\frac{A}{32 \pi s}\left(\frac{s}{4 M^{2}}\right)^{4}
$$


with specific polarization dependence and angular distribution $(S$ and $D$ waves, roughly isotropic). This wide angle elastic $\gamma \gamma$ scattering has very clear signature and small QED background. The observation of strong elastic $\gamma \gamma$ scattering raising quickly with energy will be the signal of one of these mechanisms. The study of polarization and angular dependence at photon collider and some similar processes can discriminate what mechanism is relevant.

- Effects of extra dimensions are considered in the scenario where gravity propagates in the $(4+n)$-dimensional bulk of space-time, while gauge and matter fields are confined to the $(3+1)$-dimensional world volume of a brane configuration. The extra $n$ dimensions are compactified with scale $R$ what produces the Kaluza-Klein excitations having masses $\pi n / R$. The corresponding scale in our world is assumed to be $M \sim$ few $\mathrm{TeV}$. The particles of our world interact (as $A \bar{A} \rightarrow B \bar{B}$ ) via the set of Kaluza-Klein excitations having spin 2 or 0 as e.g. $T^{\mu \nu} T^{\mu \nu} / M^{4}$, where $T^{\mu \nu}$ is stress-energy tensor. The coefficients are accumulated in the definition of $M$ (with $A \approx 1$ ).

The $\gamma \gamma$ initial state has numerical advantage as compared to $e^{+} e^{-}$one. The $\gamma \gamma$ final state has the best signature and the lowest SM background. The interference (with SM) effect enhances this anomaly for $\gamma \gamma \rightarrow W W$ process (simultaneously with enhancement of background).

- Point-like Dirac monopole 1 . This monopole existence would explain mysterious quantization of the electric charge. There is no place for it in modern theories of our world but there are no convincing reasons against its existence.

At $s \ll M^{2}$ the electrodynamics of monopoles is expected to be similar to the standard QED. At $g \sqrt{s} /(4 \pi M)<1$ (with $g=n /(2 e)$ ) the effect is described by monopole loop, and one can use coefficient $A \propto g^{8}$, calculated within QED. Both $A$ and details of angular and polarization dependence depend strongly on spin of monopole $J$, e.g., $A(J=1) / A(J=0) \approx 1900$.

Effect can be seen at TESLA500 at $M<4-10 \mathrm{TeV}$ (depending on monopole spin). Modern limitation (Tevatron) is about 10 times lower.

\section{$3 \quad$ SM and SM-like scenario}

If after experiments at LHC and $e^{+} e^{-} \mathrm{LC}$ a SM-like scenario (defined in the beginning of report) will be realized, the main problems for study at Colliders become the following:

- The study of EWSB mechanism.

- Discovery of signals of New Physics via (small) deviations from SM predictions.

- Description of observed phenomena in SM, especially QCD:

$\square$ Total cross sections, Pomeron and odderon, minijets.

$\square$ Photon structure function.

$\square$ Some problems with heavy quarks.

Photon Colliders provide new (sometimes unique) keys for solving all these problems.

\subsection{The study of EWSB}

Assuming Higgs mechanism for EWSB, one should consider 3 main opportunities:

- SM with one Higgs doublet and $M_{h}<400 \div 700 \mathrm{GeV}$ - standard SM case.

- More complex Higgs sector, the simplest variant - Two Doublet Higgs Model (2HDM).

In these cases Higgs boson will be discovered at the Tevatron or LHC, its spin and couplings to $W, Z$ and fermions will be precisely measured at $e^{+} e^{-} \mathrm{LC}$.

- SM with one Higgs doublet but with strong Higgs self-interaction. In this case standard Higgs particle does not exist and strong interaction in Higgs sector will manifest itself as the strong interaction of gauge bosons $W$ and $Z$ (their longitudinal components).

The measuring of $h \gamma \gamma$ and $h Z \gamma$ couplings is an excellent tool for these problems. Indeed,

$\square$ In the SM these couplings appear only at the loop level. Therefore, the S/B for new signals is better than that for the processes allowed at tree level.

All fundamental charged particles contribute to these effective couplings. 
The expected accuracy in the measurement of the two-photon width is $\sim 2 \%$ at the luminosity integral $30 \mathrm{fb}^{-1}$ and $M_{h} \leq 150 \mathrm{GeV} 10$. This uncertainty can be reduced with the expected 3-year luminosity integral about $500 \mathrm{fb}^{-1}$.

The anomalous interactions of Higgs boson with light 14 (CP conserving and nonconserving) can be summarized in an effective interaction

$$
h v\left(\theta_{\gamma} \frac{F_{\mu \nu} F^{\mu \nu}}{\Lambda_{\gamma}^{2}}+2 \theta_{Z} \frac{Z_{\mu \nu} F^{\mu \nu}}{\Lambda_{Z}^{2}}+i \theta_{P \gamma} \frac{F_{\mu \nu} \tilde{F}^{\mu \nu}}{\Lambda_{P \gamma}^{2}}+2 i \theta_{P Z} \frac{Z_{\mu \nu} \tilde{F}^{\mu \nu}}{\Lambda_{P Z}^{2}}\right), \quad\left(\theta_{i}=e^{i \xi_{i}}\right) .
$$

Here $F^{\mu \nu}$ and $Z^{\mu \nu}$ are standard field strengths for the electromagnetic and $Z$ field, $\tilde{F}^{\mu \nu}=$ $\varepsilon^{\mu \nu \alpha \beta} F_{\alpha \beta} / 2, v=\left(G_{F} \sqrt{2}\right)^{-1 / 2}-$ v.e.v. of Higgs field. Finally, $\xi_{i}$ are the phases of couplings, in general not equal to 0 or $\pi$.

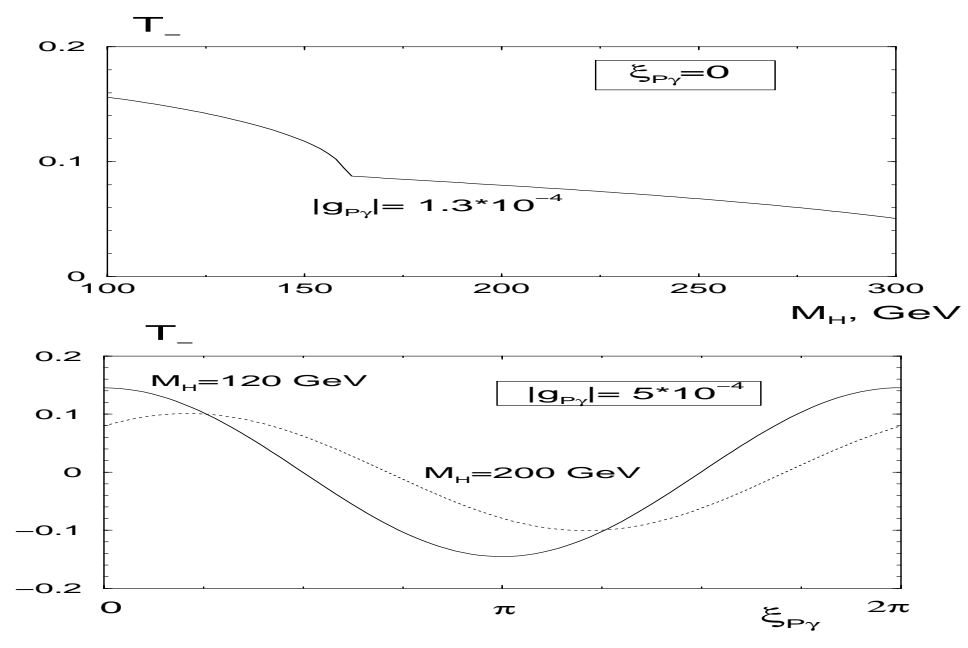

Figure 2: The longitudinal asymmetry in $\gamma \gamma \rightarrow h$ production due to anomalous interactions (3).

The CP conserving anomalies give the deviation of measured $\gamma \gamma \rightarrow h$ and $e \gamma \rightarrow e h$ cross sections from SM prediction. The CP anomalies give the polarization asymmetries - variation of cross sections with change of sign of photon helicities (longitudinal), or with the angle between directions of linear polarization of colliding photons (transverse). One example - the longitudinal asymmetry - is shown in Fig. 1 . We see that the effects can be seen at reasonable values of anomaly scales $\Lambda_{i}$ and phases $\xi_{i}$.

- Distinguishing SM/2HDM in the SM-like scenario11. The SM-like scenario can be realized both in the SM and in other models. The simplest extension of Higgs sector is the 2HDM with the Model II for the Yukawa coupling (the same is realized in MSSM). It contains 2 Higgs doublet fields $\phi_{1}$ and $\phi_{2}$ with v.e.v.'s $v \cos \beta$ and $v \sin \beta$. The physical sector contain charged scalars $H^{ \pm}$and three neutral scalars $h_{i}$ with no definite CP parity, in the CP conserving case these $h_{i}$ become scalars $h$ and $H$ (with $M_{h}<M_{H}$ ) and pseudoscalar $A$.

Generally, 2HDM permits to have relatively strong $C \mathrm{P}$ and large FCNC. To make these effects naturally weak, the terms in Higgs potential, giving $\left(\phi_{1}, \phi_{2}\right)$ mixing should be relatively small, and the properties of the observed Higgs boson are close to those of $h$ or $H$. In this case masses $M_{H}, M_{A}$ and $M_{H^{ \pm}}$are $\leq 3 \mathrm{TeV}$ due to perturbativity constraint 12 .

The SM-like scenario means that the squared coupling constants (not coupling constants themselves) are close to the SM value. In the $2 \mathrm{HDM}$ it can be realized in many ways even in the CP conserving case (Table 2). The widely discussed decoupling limit corresponds to solution $A_{h+}$ supplemented with a demand of unnatural strong $\left(\phi_{1}, \phi_{2}\right)$ mixing (giving heavy $H, A$ and $H^{ \pm}$with $M_{H} \approx M_{A} \approx M_{H^{ \pm}}$without perturbativity limitation). 


\begin{tabular}{|c|c|c|c|c|c|c|}
\hline type & notation & $\begin{array}{c}\text { observed } \\
\text { Higgs } \\
\text { boson }\end{array}$ & $\chi_{V}$ & \multicolumn{2}{|c|}{$\tan \beta$} & constraint \\
\hline \multirow{4}{*}{$\begin{array}{c}A_{\phi \pm} \\
\chi_{V} \approx \chi_{u} \approx \chi_{d}\end{array}$} & $A_{h+}$ & $\bar{h}$ & $\approx+1$ & \multirow{4}{*}{$\sqrt{\left|\frac{\epsilon_{d}}{\epsilon_{u}}\right|}$} & $\lessgtr 1$ & \multirow{4}{*}{$\epsilon_{V}=-\frac{\epsilon_{u} \epsilon_{d}}{2}$} \\
\hline & $A_{H+}$ & $\mathrm{H}$ & $\approx+1$ & & $\lessgtr 1$ & \\
\hline & $A_{h-}$ & $\mathrm{h}$ & $\approx-1$ & & $\ll 1$ & \\
\hline & $A_{H-}$ & $\mathrm{H}$ & $\approx-1$ & & $\gg 1$ & \\
\hline \multirow{2}{*}{$\begin{array}{c}B_{\phi \pm d}: \\
\chi_{V} \approx \chi_{u} \approx-\chi_{d}\end{array}$} & $B_{h+d}$ & $\mathrm{~h}$ & $\approx+1$ & \multirow{2}{*}{\multicolumn{2}{|c|}{$\sqrt{\frac{2}{\epsilon_{V}}} \gtrsim 10$}} & \multirow[t]{2}{*}{$\epsilon_{u}=-\frac{\epsilon_{V} \epsilon_{d}}{2}$} \\
\hline & $B_{H \pm d}$ & $\mathrm{H}$ & $\approx \pm 1$ & & & \\
\hline \multirow{2}{*}{$\begin{array}{c}B_{\phi \pm u}: \\
\chi_{V} \approx \chi_{d} \approx-\chi_{u}\end{array}$} & $B_{h \pm u}$ & $\mathrm{~h}$ & $\approx \pm 1$ & \multirow{2}{*}{\multicolumn{2}{|c|}{$\sqrt{\frac{\epsilon_{V}}{2}} \lesssim 0.1$}} & \multirow[t]{2}{*}{$\epsilon_{d}=-\frac{\epsilon_{V} \epsilon_{u}}{2}$} \\
\hline & $B_{H+u}$ & $\mathrm{H}$ & $\approx+1$ & & & \\
\hline \multicolumn{7}{|c|}{$\begin{array}{c}\chi_{i}=\frac{g_{i}}{g_{i}^{S M}}= \pm\left(1-\epsilon_{i}\right) \text { with } \\
i=V(\equiv Z, W) \text { or } i=u(\equiv t, c) \text { or } i=d, \ell(\equiv b, \tau) ; \quad \epsilon_{V}>0, \epsilon_{u} \epsilon_{d}<0\end{array}$} \\
\hline
\end{tabular}

Table 2: Allowed realizations of SM-like scenario in the 2HDM (II)

In these SM-like cases the observed Higgs boson can be either $h$ or $H$. The pseudoscalar $A$ and other scalar, $H$ or $h$, are almost decoupled to gauge bosons and cannot be seen at $e^{+} e^{-}$ $\mathrm{LC}$ in the standard processes. If mass of any of these elusive Higgs bosons is below $350 \mathrm{GeV}$ and $\tan \beta \ll 1$, it can be seen in $\gamma \gamma \rightarrow \gamma \gamma$ process (for $h$ - using the low energy part of photon spectrum) (and in $e^{+} e^{-} \rightarrow t \bar{t} H$ at $2 E>2 M_{t}+M_{H}$ ).

In any case, one can distinguish models via measurement of two-photon width of observed SM-like Higgs boson 11. For $M_{H^{ \pm}}=800 \mathrm{GeV}$ the ratios of the two-photon Higgs width and the of cross sections for $e \gamma \rightarrow e h$ process (for latter reaction - at $\sqrt{s}=1.5 \mathrm{TeV}$ for the left hand polarized photons) to their SM values are shown in Fig. 3 for the natural set of parameters of 2HDM. The bands around central line represent possible difference of Higgs-fermion and Higgs-
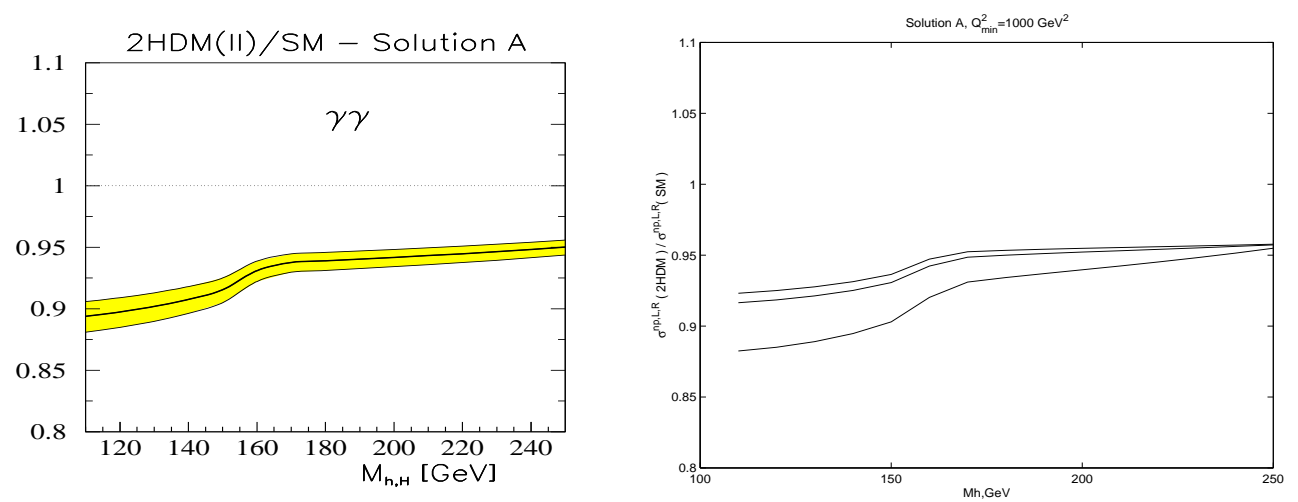

Figure 3: Solutions $A$ and $B_{\phi \pm d}$. The ratio of quantities in 2HDM to their SM values. The two-photon Higgs width - left panel; the cross section $\sigma_{L}(e \gamma \rightarrow e h)$ - right panel.

$W$ couplings (squared) from their SM values in the SM-like scenario according to anticipated uncertainty of future measurements 3 .

The deviations from SM, shown in Fig. 3 for solutions $A$ and $B_{d}$, are about $\sim 10 \%$ (one may compare with anticipated $2 \%$ accuracy). These deviations are given by contribution of heavy charged Higgs bosons. For solutions $B_{u}$ changing of relative sign of contributions of $t-$ loop and $W$-loop increases the observable cross section more than twice in comparison with SM. Therefore, measurement of two-photon width at Photon Collider can resolve these cases reliably. 
The possible strong interaction in the Higgs sector should also be the strong interaction of longitudinal $W$ 's. It is expected to be observed in the process $\gamma \gamma \rightarrow W W$. Based on the experience with process $\gamma \gamma \rightarrow \pi^{+} \pi^{-}$the following picture is expected: The strong interaction modifies weakly the cross section near the threshold in comparison with its SM value (but the phase of amplitude reproduces that of strong interacting $W_{L} W_{L}$ scattering and it can be significant). It makes strong interaction in the Higgs sector hardly observable in the cross sections below the energies, given by the masses of resonances, $1.5-2 \mathrm{TeV}$.

The charge asymmetry in the process $e \gamma \rightarrow e W^{+} W^{-}$is sensitive to this phase of amplitude even at relatively low energy of TESLA $(0.8-1 \mathrm{TeV})$ 罒, considerably below possible resonance production. Indeed, the essential contribution to this asymmetry is given by interference of two-photon production subprocess of $W W$ pair (in C-even state) and bremsstrahlung (onephoton) production subprocess (in C-odd state) like for the process $e^{+} e^{-} \rightarrow e^{+} e^{-} \pi^{+} \pi^{-} 16$. (The interference with axial part of $Z$ exchange contributes additionally to this asymmetry).

\subsection{Anomalous interactions of gauge bosons}

At relatively low energies the New Physics cannot manifest itself via new heavy particles. Thus, it reveals itself as certain anomalies in the interactions of known particles. The goal of corresponding studies is to find and discriminate these anomalies. The correlation between coefficients of different anomalies will be the key for understanding what is the nature of New Physics.

The practically unique process under interest in the $e^{+} e^{-}$mode is $e^{+} e^{-} \rightarrow W W$. With appropriate electron polarization the neutrino exchange contribution disappears, and residual cross section (described by photon and $Z$ boson exchange) has maximum about 2 pb within LEP operation interval and further decreases with energy. The cross sections of other processes with $W$ production are below $1 \mathrm{pb}$ at $\sqrt{s}<1 \mathrm{TeV}$.

At Photon Collider main processes are $\gamma \gamma \rightarrow W W$, e $\gamma \rightarrow W \nu$. Their cross sections are about $80 \mathrm{pb}$, they are independent from energy at $\sqrt{s}>200 \mathrm{GeV}$, what provides about $10^{7} W$ 's per year. Due to high value of these basic cross sections, many processes of 3-rd and 4-th order have large enough cross sections: $e \gamma \rightarrow e W W, \gamma \gamma \rightarrow Z W W, e \gamma \rightarrow \nu W Z, \gamma \gamma \rightarrow W W W W, \ldots$, see Fig. 4. Large variety of these processes allows to discover and separate well anomalies in

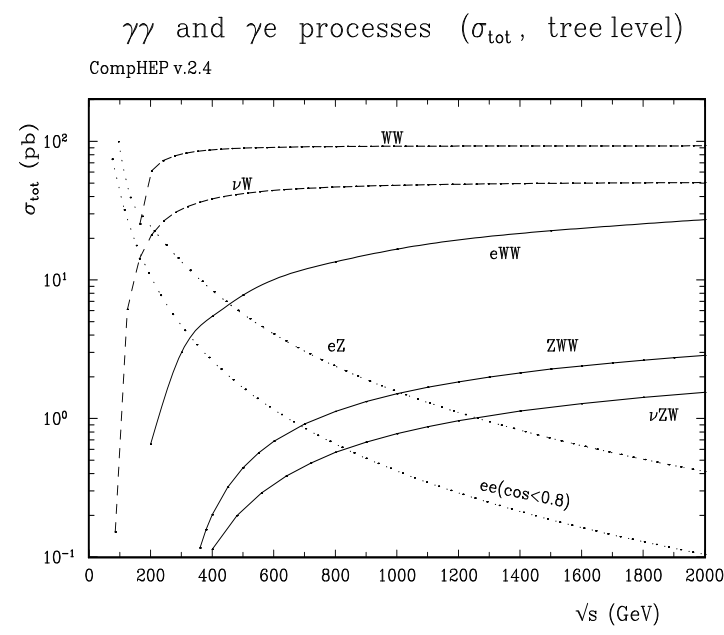

Figure 4: The 2-nd and 3-rd order processes at Photon Collider

specific processes and (or) distributions. 
The cross section $e \gamma \rightarrow \nu W \propto\left(1-2 \lambda_{e}\right)$, it is switched on or off with variation of electron helicity $\lambda_{e}$. It gives very precise test of absence of right handed currents in the interaction of $W$ with the matter. The modern simulations of process $e \gamma \rightarrow \nu W$ with only lepton decay modes for $W$ decay show that the sensitivity of this reaction to the quadruple momentum of $W$ is twice higher than it can be reached at $e^{+} e^{-} \mathrm{LC} 13$.

- The two-loop radiative corrections to $\gamma \gamma \rightarrow W W$ and $e \gamma \rightarrow \nu W$ processes should be considered. They are measurable and sensitive to the (unsolved) problems of

- construction of $S$-matrix in the theory with unstable particles;

- gluon corrections (like Pomeron exchange) between quark components of $W$ 's.

\subsection{QCD and Hadron Physics, t-quarks, etc.}

All the problems studied at HERA and LEP will be studied at Photon Collider but in the wider interval of parameters and with much higher accuracy. Among them we list those which look most interesting now.

- Nature of growth of total cross sections. The widespread concepts assume standard Regge type factorization and universal energy behavior for different processes. With Photon Colliders, - for the first time in particle physics at high enough energies - one can have the set of mass shell cross sections of very high energy processes, appropriate for the testing of factorization or its violation. That are $\sigma_{p p}$, measured at Tevatron and LHC, $\sigma_{\gamma p}$, measured at HERA, THERA and $\sigma_{\gamma \gamma}$, measurable at Photon Collider. The preliminary stage of operations with low luminosity can be used to observe cross sections at small scattering angles.

- The structure function of photon is a unique QCD object calculable completely without phenomenology impacts at high enough photon virtuality $Q^{2}$ and moderate $x$. In modern data phenomenological hadronic component of photon dominates and accuracy of data is low. The experiments at $e \gamma$ collider would increase the obtainable region of $Q^{2}$ significantly and improve accuracyl.

- The study of charge asymmetry of produced hadrons in $\gamma \gamma$ collisions will give quite new information about QCD at small distances. The charge asymmetry of the produced hadrons in the $e \gamma$ collisions with transverse momentum of scattered electron $p_{\perp} \geq 30 \mathrm{GeV}$ will show in explicit form the relation between hadron states produced by vector and axial currents 1 .

- In addition to the usually discussed problems related to the $t$ quarks, the specific one is the study of axial anomaly in the process $e \gamma \rightarrow e t \bar{t}$ existing even in the SM. At small transverse momenta of scattered electrons $p_{\perp} \rightarrow 0$ the cross section of subprocess $\gamma Z_{L} \rightarrow t \bar{t}$ with longitudinally polarized $Z$ diverges as $M_{t}^{2} / p_{\perp}^{2}$ (in contrast to $\sigma\left(\gamma \gamma_{L} \rightarrow \bar{t}\right.$ ) tending to 0 ) 1 .

- The study of charge asymmetry in $e \gamma \rightarrow e b \bar{b}$ can help to discover $\mathrm{S}$ and $\mathrm{D}$ wave resonances in $b \bar{b}$.

\section{The using of conversion region}

The $e \rightarrow \gamma$ conversion region is $e \gamma$ collider with c.m.s. energy about $1.2 \mathrm{MeV}$ and with huge luminosity about $0.1 \mathrm{fb}^{-1} / \mathrm{sec!}$ It will be a unique source of light Goldstone particles (axions, majorons, etc. - LGP, a) 19 , weakly interacting with the matter. They are expected to exist in numerous schemes 20 .

The production processes are $e+\gamma_{0} \rightarrow e+a$ and $\gamma \gamma_{0} \rightarrow a$ (here $\gamma_{0}$ denotes laser photon). Denoting $x=4 E \omega_{0} / m_{e}^{2}$ and $a=m_{a}^{2} / m_{e}^{2}$, we obtain that the LGP energy in these reactions is limited from above as

$$
E_{a} \leq \frac{x+a+\sqrt{(x-a)^{2}-4 a}}{2(x+1)} E \text { for } e \gamma_{0} \rightarrow e a, \text { or } E_{a}=\frac{m_{a}^{2}}{4 \omega_{0}} \leq \frac{x}{x+1} E \text { for } \gamma \gamma_{0} \rightarrow a .
$$


The angular spread of LGP is very narrow, in practice, it is given by angular spread of incident electrons within beam $\left(\sim 10^{-5}\right)$. Note that due to high density of laser photon in the conversion region the nonlinear processes like $e+n \gamma_{0} \rightarrow e+a(n=2,3, .$.$) , etc., also become possible. The$ upper limit of LGP energy in these reactions is higher than that given by eq. (四).

Some numerical estimates for LGP being axion or arion with mass about $10 \mathrm{KeV}$ and electron beam energy $250 \mathrm{GeV}$ are presented in Table 3 (the production rate of LGP's familon and majoron is negligibely low).

\begin{tabular}{|c|c|c|c|}
\hline & $g_{a e e}$ & Cross-section $\sigma\left(\mathrm{cm}^{2}\right)$ & The number of produced LGP's per year \\
\hline Standard axion & $2 \cdot 10^{-6}$ & $2.2 \cdot 10^{-35}$ & $2 \cdot 10^{10}$ \\
\hline "Invisible" axion & $3 \cdot 10^{-8}$ & $4.9 \cdot 10^{-39}$ & $5 \cdot 10^{6}$ \\
\hline Arion & $2 \cdot 10^{-6}$ & $2 \cdot 10^{-35}$ & $2 \cdot 10^{10}$ \\
\hline
\end{tabular}

Table 3: Estimates of typical coupling constants, production cross sections and the number of LGP's, produced per year.

To observe these LGP's, the special detector is proposed. It should be some pin-type lead rod with radius about $2 \mathrm{~cm}$ and length $300-500 \mathrm{~m}$, placed in vacuum behind a shield to get rid of the background (Fig. 5). The LGP interacts with lead nuclei like pion but with much lower coupling constant, $a+P b \rightarrow h$ (hadrons). The produced hadron jets with total energy $\sim \epsilon_{a}$ and characteristic transverse momentum $p_{\perp} \sim 300 \mathrm{MeV} / \mathrm{c}$ should be recorded in the round scinillator and calorimeter with diameter in 1-3 $\mathrm{m}$ in the end of the device.

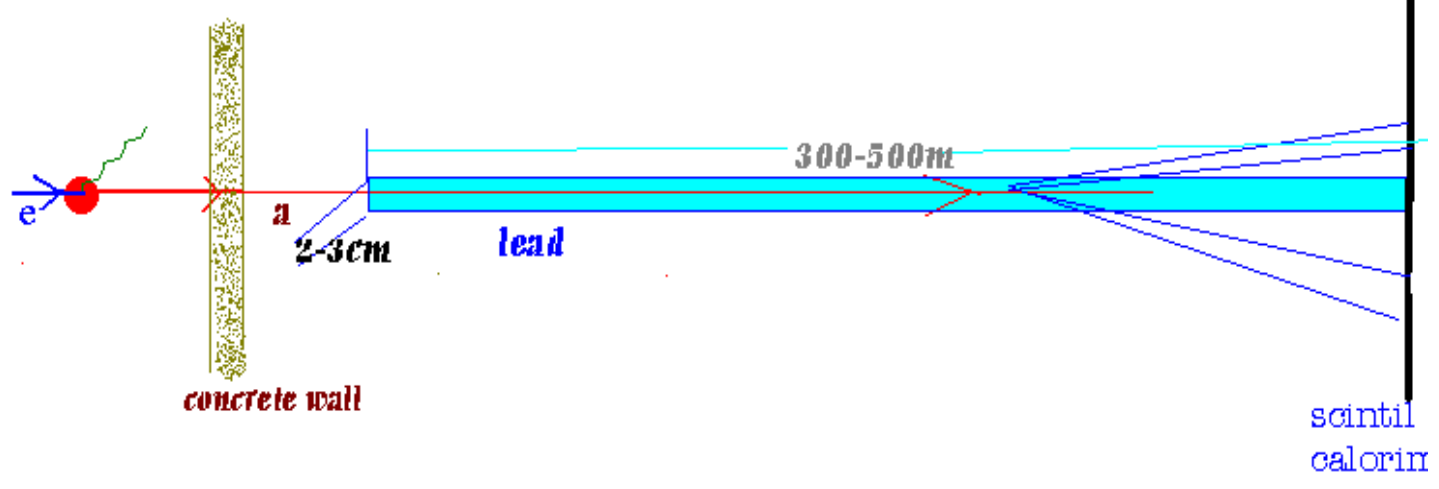

Figure 5: Scheme of LGP detection

The main background is given by neutrinos produced by photons in the shield (reactions like $\gamma+p \rightarrow \pi+\ldots, \pi \rightarrow \mu \nu)$. The proposed scheme allows to reduce this background strongly. Indeed, the energy of the main part of such neutrinos is much lower than the upper limit (4). Besides, the produced neutrinos are spread over the angular interval which is about 3-4 orders of magnitude wider than that for LGP's, reducing relative flux of neutrino in the lead rod by factor $10^{-6}-10^{-8}$.

\section{Acknowledgments}

We are thankful D. Anipko, V.Ilyin, I. Ivanov, M. Krawczyk, P. Olsen, A. Pak, M. Vychugin for collaboration related different parts of paper and A. Djouadi, V. Serbo, M. Spira, V. Telnov, P.Zerwas for useful discussions. This work was supported by RFBR grants 99-02-17211 and 0015-96691 and INTAS grant 00-00679. I.F.G. is also thankful by organizers of Eriche Workshop 
for invitation and support.

\section{References}

1. I.F. Ginzburg, G.L. Kotkin, V.G. Serbo, V.I. Telnov, Nucl. Instrum. Methods 205 (1983) 47; I.F. Ginzburg, G.L. Kotkin, S.L. Panfil, V.G. Serbo, V.I. Telnov, Nucl. Instrum. Methods A 219 (1984) 5; Zeroth-order Design Report for the NLC, SLAC Report 474 (1996); R. Brinkmann et al., Nucl. Instrum. Methods 406 (1998) 13.

2. B.Badelek et al. TESLA Technical Design Report, p. VI, chap.1 DESY 2001-011, TESLA Report 2001-23, TESLA FEL 2001-05 (2001) hep-ex/0108012, p.1-98

3. R.D. Heuer et al. TESLA Technical Design Report, p. III DESY 2001-011, TESLA Report 2001-23, TESLA FEL 2001-05 (2001) 192p. hep-ph/0106315

4. E. Boos, A. De Roek, I.F. Ginzburg, K. Hagiwara, R.D. Heuer, G. Jikia, J. Kwiecinski, D.J. Miller, T. Takahashi, V.I. Telnov, T. Rizzo, I. Watanabe, P.M. Zerwas, Goldplated processes at photon collider, Nucl. Instrum. Methods A 472 (2001) 100-120; hep-ph/0103090

5. S.P. Li, H.C. Liu, D. Silverman Phys. Rev. D 31 (1987) 1736.

6. D.S. Gorbunov, V.A. Ilyin, hep-ph/0004092; D.S. Gorbunov, V.A. Ilyin, V.I. Telnov, hep$\mathrm{ph} / 0012175$

7. See e.g. H. Davoudiasi J. Mod. Phys. A 15 (2000) 2613; K. Cheung, Phys. Rev. D 61 (2000) 0105015; T. Rizzo, Phys. Rev. D 60 (1999) 115010, hep-ph/0008037

8. I.F. Ginzburg, S.L. Panfil, Sov. Yad. Fiz. 36 (1982) 850; I.F. Ginzburg, A. Schiller, Phys. Rev. D 60 (1999) 075016.

9. E. Witten, Nucl. Phys. B 12 (1977) 189.

10. G. Jikia, S. Söldner-Rembold, Nucl. Phys. B (Proc. Suppl.) 82 (2000) 373; M. Melles, W.J. Stirling, V.A. Khoze,Phys. Rev. D61(2000) 054015.

11. I.F. Ginzburg, M. Krawczyk, P.Osland, hep-ph/9909455; hep-ph/0101229; hep$\mathrm{ph} / 0101331$.

12. I.F. Ginzburg, M. Krawczyk, P.Osland, in preparation; I.F. Ginzburg, M.V. Vychugin, hep-ph/0201117.

13. D.A. Anipko, I.F. Ginzburg, A.V. Pak, hep-ph/0201072.

14. A.T. Banin, I.F. Ginzburg, I.P. Ivanov, Phys. Rev. B59 (1999) 115001; I.F. Ginzburg, I.P. Ivanov, Eur. Phys. Journ. C 22 (2001) 411-421, hep-ph/0004069; E. Gabrieli, V.A. Ilyin, B. Mele, Phys. Rev. D 60 (1999) 113005

15. I.F. Ginzburg, Proc. 9th Int. Workshop on Photon - Photon Collisions, San Diego (1992) 474-501, World Sc. Singapore.

16. V.L. Chernyak, V.G. Serbo, Nucl. Phys. B 67 (1973) 464; I.F. Ginzburg, A. Schiller, V.G. Serbo, Eur. Phys. Journ. C18 (2001) 731.

17. I.F. Ginzburg, to be published in Proc. PHOTON'2001

18. I.F. Ginzburg, V.A. Ilyin, in preparation.

19. S.I. Polityko, Sov. Yad. Fiz. 43 (1986) 146; 56 (1993) 144; J.Phys.G: Nucl.Part.Phys. 27 (2001) 2421.

20. See e.g., P.Sikivie, hep-ph/0002154 